\title{
ON THE EXISTENCE, UNIQUENESS AND PARAMETRIC DEPENDENCE ON THE COEFFICIENTS OF THE SOLUTION PROCESSES IN McSHANE'S STOCHASTIC INTEGRAL EQUATIONS
}

\author{
ADRIAN CONSTANTIN
}

\begin{abstract}
In this paper we use the Schauder fixed point theorem and methods of integral inequalities in order to prove a result on the existence, uniqueness and parametric dependence on the coefficients of the solution processes in McShane stochastic integral equations
\end{abstract}

\section{Introduction}

In this paper we consider the problem of the existence and uniqueness of the solution processes in the stochastic integral system

$$
\begin{aligned}
& \text { (1) } x^{i}(t)=\alpha^{i}(t)+\sum_{j=1}^{r} \int_{0}^{t} g_{j}^{i}(s, x(s)) d z_{j}(s) \\
& +\sum_{j, k=1}^{r} \int_{0}^{t} h_{j k}^{i}(s, x(s)) d z_{j}(s) d z_{k}(s), \quad 0 \leq t \leq a, \quad i=1, \ldots, n,
\end{aligned}
$$

where the stochastic integrals involving $d z_{j}$ are interpreted as McShane stochastic integrals.

Some previous results were given by McShane [13] in the special case in which $\alpha^{i}$ is not depending on time and the processes $z_{j}$ are sample continuous and by Elworthy [8] which does not require these conditions but considers stronger hypotheses on $g_{j}^{i}$ and $h_{j k}^{i}$. Recently, Angulo Ibañez and Gutiérrez Jaimez [1] proved (under weaker requirements than in $[\mathbf{8}]$ and $[\mathbf{1 3}]$ ) an existence and uniqueness theorem for (1) assuming 
that the functions $g_{j}^{i}$ and $h_{j k}^{i}$ satisfy a Lipschitz condition and the processes $z_{j}$ satisfy a $K$-condition, using an adequate version of the FominKolmogorov fixed point theorem. They considered in [2] the problem of the convergence of the solution processes in McShane's stochastic integral equation systems with coefficients depending on a parameter under similar assumptions to [1].

Under the hypothesis of a weaker condition than the Lipschitz condition on $g_{j}^{i}$ and $h_{j k}^{i}$ we prove an existence and uniqueness result for the solution process of equation (1) applying Shauder's fixed point theorem $[\mathbf{1 5}]$ and methods of integro-differential inequalities (see [3], [4], [5], [7], [9]). Under the same hypothesis we prove also the continuity with respect to the initial condition and we consider the problem of parametric dependence of the solution processes on the coefficients, generalizing the results of [2].

\section{Preliminaries}

Let $(\Omega, F, P)$ be a complete probability space and let $\left\{F_{t}, 0 \leq t \leq a\right\}$ be a family of complete $\sigma$-subalgebras of $F$ such that if $0 \leq s \leq t \leq a$ then $F_{s} \subseteq F_{t}$.

Let $L_{2}$ be the space of all random variables $y: \Omega \rightarrow \mathbb{R}$ with finite $L_{2}$-norm $\|\cdot\|$ and let $L_{2}^{n}$ be the space of all random variables $x: \Omega \rightarrow \mathbb{R}^{n}$ with finite norm $\|\cdot\|_{n}$,

$$
\|x\|_{n}^{2}=\sum_{i=1}^{n}\left\|x_{i}\right\|^{2}, \quad x=\left(x_{1}, \ldots, x_{n}\right) \in L_{2}^{n} .
$$

We say that the real valued second order stochastic process $z$ on $[0, a]$ satisfies a $K$-condition if $z$ is adapted to the $F_{t}$ (i.e. $z(t)$ is $F_{t}$-measurable for every $t \in[0, a])$ and

$$
\left|E\left[(z(t)-z(s))^{p} / F_{s}\right]\right| \leq K|t-s|
$$

a.s. whenever $0 \leq s \leq t \leq a, p=1,2,4$. An example of such a process is a Wiener process with respect to the $F_{t}, 0 \leq t \leq a$.

Let us define also for each second order process $x$ on $[0, a]$ the norm

$$
\|\| x \|=\sup _{t \in[0, a]}\{\|x(t)\|\} .
$$

For the basic elements of the McShane stochastic calculus theory we refer to McShane [13] and Elworthy [8]. Let us remind only that if $f$ : 
$[0, a] \rightarrow L_{2}$ is a measurable process adapted to the $F_{t}$ and if $t \rightarrow\|f(t)\|^{2}$ is Lebesgue integrable on $[0, a]$, then $([\mathbf{8}],[\mathbf{9}])$, if $z_{1}$ and $z_{2}$ satisfy a $K$-condition, the McShane integrals

$$
\int_{0}^{a} f(s) d z_{1}(s), \quad \int_{0}^{a} f(s) d z_{1}(s) d z_{2}(s)
$$

exist, and the following estimates are true

$$
\begin{aligned}
\left\|\int_{0}^{a} f(s) d z_{1}(s)\right\| & \leq C\left\{\int_{0}^{a}\|f(s)\|^{2} d s\right\}^{1 / 2} \\
\left\|\int_{0}^{a} f(s) d z_{1}(s) d z_{2}(s)\right\| & \leq C\left\{\int_{0}^{a}\|f(s)\|^{2} d s\right\}^{1 / 2}
\end{aligned}
$$

where $C=(2+8 K a)^{1 / 2}$.

We will use in this paper the following result

Lemma (Bihari [3]). Let $v, h$ be positive, continuous functions on $0 \leq t \leq a$, and let $q$ be a nonnegative constant; further let $w$ be a positive nondecreasing function on $[0, \infty)$. Then the inequality

$$
v(t) \leq q+\int_{0}^{t} h(s) w(v(s)) d s, \quad 0 \leq t \leq a,
$$

implies the inequality

$$
v(t) \leq G^{-1}\left(G(q)+\int_{0}^{t} h(s)\right) d s, \quad 0 \leq t \leq a^{\prime},
$$

where $G(u)=\int_{1}^{u} \frac{d s}{w(s)}, u>0$, and $a^{\prime}$ is defined so that $G(q)+\int_{0}^{t} h(s) d s$ lies within the domain of definition of $G^{-1}$ for $0 \leq t \leq a^{\prime}$.

For proofs of the lemma, see [3] and [5].

\section{Existence and uniqueness of solutions}

Let $C[0, a]$ denote the space of all processes $x:[0, a] \rightarrow L_{2}^{n}$ which are continuous and adapted to the $F_{t}$. A solution to the equation (1) on $[0, a]$ is a process $x \in C[0, a]$ which satsifies $(1)$ on $[0, a]$.

Let us assume that

$\left(\mathrm{H}_{1}\right)$ the noise processes $z_{j}, j=1, \ldots, r$, satisfy on $[0, a]$ a $K$-condition; 
$\left(\mathrm{H}_{2}\right)$ if $f$ is any one of the functions $g_{j}^{i}, h_{j k}^{i}:[0, a] \times L_{2}^{n} \rightarrow L_{2}, i=$ $1, \ldots, n ; j, k=1, \ldots, r$, then $f(s, x)$ is continuous in $x$ on $L_{2}^{n}$ for every $s \in[0, a]$, and for any $x \in C[0, a]$, the process $t \rightarrow f(t, x(t))$ is measurable and $F_{t}$-adapted with $t \rightarrow\|f(t, x(t))\|^{2}$ bounded on $[0, a]$

$\left(\mathrm{H}_{3}\right)$ there exists a continuous, nondecreasing function $w: \mathbb{R}_{+} \rightarrow \mathbb{R}_{+}$ with $w(0)=0, w(t)>0$ for $t>0$ and

$$
\lim _{t \rightarrow 0} \int_{t}^{1} \frac{d s}{w(s)}=\infty, \quad \lim _{t \rightarrow \infty} \int_{1}^{t} \frac{d s}{w(s)}=\infty
$$

such that

$$
\|f(t, x)-f(t, y)\|^{2} \leq w\left(\|x-y\|_{n}^{2}\right), \quad t \in[0, a], \quad x, y \in L_{2}^{n}
$$

$\left(\mathrm{H}_{4}\right)$ the initial condition $\alpha$ belongs to $C[0, a]$.

Theorem 1. Let us suppose that the hypotheses $\left(\mathrm{H}_{1}\right)-\left(\mathrm{H}_{4}\right)$ are satisfied. Then there exists an unique solution of the equation (1) on $[0, a]$.

Proof: Let us first prove the existence of a solution on $[0, a]$.

We define the operator $T: C[0, a] \rightarrow C[0, a]$ by

$$
\begin{aligned}
T x(t)=\alpha(t)+\sum_{j=1}^{r} & \int_{0}^{t} g_{j}(s, x(s)) d z_{j}(s) \\
& +\sum_{j, k=1}^{r} \int_{0}^{t} h_{j k}(s, x(s)) d z_{j}(s) d z_{k}(s), \quad 0 \leq t \leq a .
\end{aligned}
$$

Let $M=2 n \sup _{t \in[0, a] \|}\|\alpha(t)\|_{n}^{2}+4 n C^{2}\left(r+r^{2}\right)^{2} K a$ where

$$
K=\max \left\{\sup _{\substack{t \in[0, a] \\ i=1, \ldots, n ; j=1, \ldots, r}}\left\|g_{j}^{i}(t, 0)\right\|^{2}, \quad \sup _{\substack{t \in[0, a] \\ i=1, \ldots, n ; j, k=1, \ldots, r}}\left\|h_{j k}^{i}(t, 0)\right\|^{2}\right\}
$$

and let us consider

$$
G:(0, \infty) \rightarrow \mathbb{R}, \quad G(u)=\int_{1}^{u} \frac{d s}{w(s)}
$$

(the hypotheses guarantee that $G$ is a bijection from $(0, \infty)$ to $\mathbb{R}$ ). 
We also consider the function

$$
m(t)=G^{-1}\left(G(M)+4 n C^{2}\left(r+r^{2}\right)^{2} t\right), \quad 0 \leq t \leq a .
$$

This function has the property that

$$
m(t)=M+4 n C^{2}\left(r+r^{2}\right)^{2} \int_{0}^{t} w(m(s)) d s, \quad 0 \leq t \leq a,
$$

being the solution of the differential equation

$$
m^{\prime}(t)=4 n C^{2}\left(r+r^{2}\right)^{2} w(m(t)), \quad 0 \leq t \leq a,
$$

with initial condition $m(0)=M$.

We consider the set

$$
B=\left\{x \in C[0, a]:\|x(t)\|_{n}^{2} \leq m(t), \quad 0 \leq t \leq a\right\} .
$$

This is a closed, bounded and convex subset of the Banach space $C[0, a]$ and we will show that $T(B) \subset B$.

Let $x \in B$. We have then that

$$
\begin{aligned}
& \left\|\int_{0}^{t} g_{j}^{i}(s, x(s)) d z_{j}(s)\right\| \leq C\left\{\int_{0}^{t}\left\|g_{j}^{i}(s, x(s))\right\|^{2} d s\right\}^{1 / 2} \\
& \leq C\left\{\int_{0}^{t}\left(\left\|g_{j}^{i}(s, x(s))-g_{j}^{i}(s, 0)\right\|+\left\|g_{j}^{i}(s, 0)\right\|\right)^{2} d s\right\}^{1 / 2} \\
& \leq C\left\{\int_{0}^{t}\left(2\left\|g_{j}^{i}(s, x(s))-g_{j}^{i}(s, 0)\right\|^{2}+2\left\|g_{j}^{i}(s, 0)\right\|^{2}\right) d s\right\}^{1 / 2} \\
& \leq C\left\{2 \int_{0}^{t} w\left(\|x(s)\|_{n}^{2}\right) d s+2 K t\right\}^{1 / 2}, \quad 0 \leq t \leq a,
\end{aligned}
$$

and similarly

$$
\begin{aligned}
\| \int_{0}^{t} h_{j k}^{i}(s, x(s)) & d z_{j}(s) d z_{k}(s) \| \\
\leq C & \left\{2 \int_{0}^{t} w\left(\|x(s)\|_{n}^{2}\right) d s+2 K t\right\}^{1 / 2}, 0 \leq t \leq a .
\end{aligned}
$$

We deduce that

$$
\begin{aligned}
\|T x(t)\|_{n} & \leq \sqrt{n} \sup _{t \in[0, a]}\|\alpha(t)\|_{n} \\
& +\sqrt{2 n}\left(r+r^{2}\right) C\left\{\int_{0}^{t} w\left(\|x(s)\|_{n}^{2}\right) d s+K t\right\}^{1 / 2}, \quad 0 \leq t \leq a,
\end{aligned}
$$


thus (since $x \in B$ )

$$
\begin{aligned}
& \|T x(t)\|_{n}^{2} \\
& \leq\left\{\sup _{t \in[0, a]} \sqrt{n}\|\alpha(t)\|_{n}+\sqrt{2 n}\left(r+r^{2}\right) C\left\{\int_{0}^{t} w(m(s)) d s+K a\right\}^{1 / 2}\right\}^{2} \\
& \leq 2 n \sup _{t \in[0, a]}\|\alpha(t)\|_{n}^{2}+4 n C^{2}\left(r+r^{2}\right)^{2} \int_{0}^{t} w(m(s)) d s+4 n C^{2}\left(r+r^{2}\right)^{2} K a \\
& =M+4 n C^{2}\left(r+r^{2}\right)^{2} \int_{0}^{t} w(m(s)) d s=m(t), \quad 0 \leq t \leq a .
\end{aligned}
$$

Hence $T(B) \subset B$.

In a similar way we prove that if $x \in B$, then

$$
\begin{array}{r}
\|T x(t)-T x(s)\|_{n}^{2} \leq 4 n C^{2}\left(r+r^{2}\right)^{2} \int_{s}^{t} w(m(u)) d u+2\|\alpha(t)-\alpha(s)\|_{n}^{2} \\
+4 n C^{2}\left(r+r^{2}\right)^{2}(t-s) K, \quad 0 \leq s \leq t \leq a
\end{array}
$$

from where we conclude that the set $T(B)$ is equicontinuous.

On the other hand we have for $x, y \in B$ that

$$
\begin{aligned}
& \left\|T x^{i}(t)-T y^{i}(t)\right\| \leq C \sum_{j=1}^{r}\left\{\int_{0}^{t}\left\|g_{j}^{i}(s, x(s))-g_{j}^{i}(s, y(s))\right\|^{2} d s\right\}^{1 / 2} \\
& +C \sum_{j, k=1}^{r}\left\{\int_{0}^{t}\left\|h_{j k}^{i}(s, x(s))-h_{j k}^{i}(s, y(s))\right\|^{2} d s\right\}^{1 / 2}, \quad 0 \leq t \leq a .
\end{aligned}
$$

From $\left(\mathrm{H}_{3}\right)$ and the continuity of $g_{j}^{i}(s, x)$ and $h_{j k}^{i}(s, x)$ in $x$ we deduce by the Lebesgue convergence theorem that $T$ is continuous. An application of Schauder's fixed point theorem enables us to deduce that $T$ has a fixed point in $B$, thus equation (1) has a solution on $[0, a]$.

Let us now prove the uniqueness of solutions for equation (1).

Suppose that there exist two different solutions $x, y \in C\left[0, a_{1}\right]$ of equation (1) on some interval $\left[0, a_{1}\right]$ with $0<a_{1} \leq a$. Then there exist points $0<t \leq a_{1}$ with $\|x(t)-y(t)\|_{n}>0$. Let $b$ be the lower bound of these $t$. We have then that $\|x(b)-y(b)\|_{n}=0$, but $\|x(t)-y(t)\|_{n}>0$ for $b<t \leq b+\beta$ with a certain number $\beta>0$. 
We have that

$$
\begin{aligned}
\| x^{i}(t)- & y^{i}(t) \| \leq \sum_{j=1}^{r} C\left\{\int_{0}^{t}\left\|g(s, x(s))-g_{j}^{i}(s, y(s))\right\|^{2} d s\right\}^{1 / 2} \\
& +\sum_{j, k=1}^{r} C\left\{\int_{0}^{t}\left\|h_{j k}^{i}(s, x(s))-h_{j k}^{i}(s, y(s))\right\|^{2} d s\right\}^{1 / 2} \\
& \leq\left(C r+C r^{2}\right)\left\{\int_{0}^{t} w\left(\|x(s)-y(s)\|_{n}^{2}\right) d s\right\}^{1 / 2}, \quad 0 \leq t \leq a_{1},
\end{aligned}
$$

thus

$$
\|x(t)-y(t)\|_{n}^{2} \leq n\left(C r+C r^{2}\right)^{2} \int_{0}^{t} w\left(\|x(s)-y(s)\|_{n}^{2}\right) d s, \quad 0 \leq t \leq a_{1} .
$$

Since $w(0)=0$ and $\|x(t)-y(t)\|_{n}=0$ on $[0, b]$ we obtain that

$$
\|x(t)-y(t)\|_{n}^{2} \leq n\left(C r+C r^{2}\right)^{2} \int_{b}^{t} w\left(\|x(s)-y(s)\|_{n}^{2}\right) d s, \quad b \leq t \leq b+\beta,
$$

thus (denoting $v(t)=\|x(t)-y(t)\|_{n}^{2}, b \leq t \leq b+\beta$ ) for every $\varepsilon>0$ we have

$$
v(t) \leq \varepsilon+n\left(C r+C r^{2}\right)^{2} \int_{b}^{t} w(v(s)) d s, \quad b \leq t \leq b+\beta .
$$

Let $V_{\varepsilon}(t)=\varepsilon+n\left(C r+C r^{2}\right)^{2} \int_{b}^{t} w(v(s)) d s, b \leq t \leq b+\beta$. We have then that

$$
V_{\varepsilon}^{\prime}(t)=n\left(C r+C r^{2}\right)^{2} w(v(t)) \leq n\left(C r+C r^{2}\right)^{2} w\left(V_{\varepsilon}(t)\right), \quad b \leq t \leq b+\beta,
$$

thus (since $V_{\varepsilon}(t)>0$ on $[b, b+\beta]$ )

$$
\frac{V^{\prime}(t)}{w\left(V_{\varepsilon}(t)\right)} \leq n\left(C r+C r^{2}\right)^{2}, \quad b \leq t \leq b+\beta .
$$

An integration yields

$$
G\left(V_{\varepsilon}(t)\right)-G\left(V_{\varepsilon}(b)\right) \leq n\left(C r+C r^{2}\right)^{2}(t-b), \quad b \leq t \leq b+\beta,
$$

thus

$$
\begin{aligned}
G\left(V_{\varepsilon}(t)\right) \leq G\left(V_{\varepsilon}(b)\right)+n( & \left.C r+C r^{2}\right)^{2}(t-b) \\
& \leq G(\varepsilon)+n\left(C r+C r^{2}\right)^{2}, \quad b \leq t \leq b+\beta .
\end{aligned}
$$


Since $0<v(t) \leq V_{\varepsilon}(t)$ for $b<t \leq b+\beta$, we deduce that

$$
G(v(t)) \leq G(\varepsilon)+n\left(C r+C r^{2}\right)^{2} \beta, \quad b<t \leq b+\beta,
$$

for every $\varepsilon>0$.

This leads to a contradiction since $v(t)>0$ for $t \in(b, b+\beta]$ thus $G(v(t))$ is a real number for $b<t \leq b+\beta$, but $\lim _{\varepsilon \rightarrow 0} G(\varepsilon)=-\infty$.

Thus equation (1) has an unique solution on $[0, a]$.

This completes the proof of Theorem 1.

Remark 1. Theorem 1 is not only an existence and uniqueness result, it provides also a bound for the solution $x \in C[0, a]$ :

$$
\|x(t)\|_{n}^{2} \leq m(t), \quad 0 \leq t \leq a .
$$

Remark 2. If the hypothesis $\left(\mathrm{H}_{3}\right)$ is satisfied with $w(t)=L t$ on $\mathbb{R}_{+}$ ( $L>0$ being a real constant) we obtain the existence and uniqueness theorem of Angulo Ibañez and Gutiérrez Jaimez [1] and as a special case (when $\alpha$ is not depending on time $t$ and the processes $z_{j}$ are sample continuous) of this we obtain the existence and uniqueness result of McShane [13]. Our requirements are weaker in some aspects with respect to those in [8] (Elworthy requires in [8] Lipschitz conditions on the functions $g_{j}^{i}$ and $\left.h_{j k}^{i}\right)$.

\section{Continuity with respect to the initial condition}

We proved in the preceding section that if the conditions $\left(\mathrm{H}_{1}\right)-\left(\mathrm{H}_{3}\right)$ are satisfied, the stochastic integral equations

$$
\begin{aligned}
x(t)=\alpha_{k}(t)+ & \sum_{j=1}^{r} \int_{0}^{t} g_{j}(s, x(s)) d z_{j}(s) \\
& +\sum_{j, k=1}^{r} \int_{0}^{t} h_{j k}(s, x(s)) d z_{j}(s) d z_{k}(s), \quad 0 \leq t \leq a,
\end{aligned}
$$

have an unique solution $x_{k} \in C[0, a]$ if $\alpha_{k} \in C[0, a], k \geq 1$.

Theorem 2. If the hypotheses $\left(\mathrm{H}_{1}\right)-\left(\mathrm{H}_{3}\right)$ are satisfied and if $\alpha_{1}, \alpha_{2} \in$ $C[0, a]$, then

$$
\|\left.\left|x_{1}-x_{2}\right|\right|_{n} ^{2} \leq G^{-1}\left(G\left(K_{1}\left\|\left|\alpha_{1}-\alpha_{2}\right|\right\|_{n}^{2}\right)+K_{2}\right)
$$


where $K_{1}=1+r+r^{2}$ and $K_{2}=n K_{1}\left(C^{2} r+C^{2} r^{2}\right) a$.

Proof: In a similar way to the proof of Theorem 1 we can state

$$
\begin{aligned}
& \left\|x_{1}^{i}(t)-x_{2}^{i}(t)\right\|^{2} \\
& \leq\left\{\left\|\alpha_{1}^{i}(t)-\alpha_{2}^{i}(t)\right\|+\sum_{j=1}^{r} C\left\{\int_{0}^{t}\left\|g_{j}^{i}\left(s, x_{1}(s)\right)-g_{j}^{i}\left(s, x_{2}(s)\right)\right\|^{2} d s\right\}^{1 / 2}\right. \\
& \left.+\sum_{j, k=1}^{r} C\left\{\int_{0}^{t}\left\|h_{j k}^{i}\left(s, x_{1}(s)\right)-h_{j k}^{i}\left(s, x_{2}(s)\right)\right\|^{2} d s\right\}^{1 / 2}\right\}^{2} \\
& \leq\left(1+r+r^{2}\right)\left\{\left\|\alpha_{t}^{i}(t)-\alpha_{2}^{i}(t)\right\|^{2}+\sum_{j=1}^{r} C^{2} \int_{0}^{t} w\left(\left\|x_{1}(s)-x_{2}(s)\right\|_{n}^{2}\right) d s\right. \\
& \left.+\sum_{j, k=1}^{r} C^{2} \int_{0}^{t} w\left(\left\|x_{1}(s)-x_{2}(s)\right\|_{n}^{2}\right) d s\right\}, \quad 0 \leq t \leq a,
\end{aligned}
$$

thus (taking supremes on $[0, a]$ to the initial condition term)

$$
\begin{aligned}
& \left\|x_{1}(t)-x_{2}(t)\right\|_{n}^{2} \leq\left(1+r+r^{2}\right) \mid\left\|\alpha_{1}-\alpha_{2}\right\|_{n}^{2} \\
+ & n\left(1+r+r^{2}\right)\left(C^{2} r+C^{2} r^{2}\right) \int_{0}^{t} w\left(\left\|x_{1}(s)-x_{2}(s)\right\|_{n}^{2}\right) d s, \quad 0 \leq t \leq a .
\end{aligned}
$$

By Bihari's inequality we obtain that

$$
\begin{aligned}
\left\|x_{1}(t)-x_{2}(t)\right\|_{n}^{2} \leq G^{-1} & \left(G \left(\left(1+r+r^{2}\right)\|\| \alpha_{1}-\alpha_{2} \|_{n}^{2}\right.\right. \\
& \left.+n\left(1+r+r^{2}\right)\left(C^{2} r+C^{2} r^{2}\right) t\right), \quad 0 \leq t \leq a,
\end{aligned}
$$

so that

$$
\left\|\left|x_{1}-x_{2}\right|\right\|_{n}^{2} \leq G^{-1}\left(G\left(K_{1}\left\|\mid \alpha_{1}-\alpha_{2}\right\| \|_{n}^{2}\right)+K_{2}\right) .
$$

Theorem 3. If the hypotheses $\left(\mathrm{H}_{1}\right)-\left(\mathrm{H}_{3}\right)$ are satisfied, $\alpha, \alpha_{n} \in C[0, a]$ and $\lim _{k \rightarrow \infty} \mid\left\|\alpha_{k}-\alpha\right\| \|_{n}=0$, we have that

$$
\lim _{k \rightarrow \infty}\left\||| x_{k}-x \mid\right\|_{n}=0
$$

where $x \in C[0, a]$ is the solution of equation (1) and $x_{k}$ is the solution of (2.k) with initial condition $\alpha_{k} \in C[0, a], k \geq 1$.

Proof: By Theorem 2 we have that

$$
\left\|\left|x_{k}-x\right|\right\|_{n}^{2} \leq G^{-1}\left(G\left(K_{1}\left|\left\|\alpha_{k}-\alpha \mid\right\|^{2}\right)+K_{2}\right)\right.
$$


thus

$$
G\left(\left.||\left|x_{k}-x\right|\right|_{n} ^{2}\right) \leq G\left(K_{1}\left|\left\|\alpha_{k}-\alpha \mid\right\|^{2}\right)+K_{2} .\right.
$$

Since $\lim _{u \rightarrow 0} G(u)=-\infty$ and $\lim _{k \rightarrow \infty}\left\|\mid \alpha_{k}-\alpha\right\|_{n}^{2}=0$ we deduce that

$$
\lim _{k \rightarrow \infty} G\left(\|\| x_{k}-x \mid \|_{n}^{2}\right)=-\infty
$$

thus $\lim _{k \rightarrow \infty}\left\||| x_{k}-x \mid\right\|_{n}=0$.

Remark 3. It seems (see the Introduction to [2]) that the first who considered the problem of the continuous dependence of the solution process with respect to the initial condition in the context of McShane's stochastic integrals were Angulo Ibañez and Gutiérrez Jaimez [2]. Our hypotheses do require a weaker condition than the Lipschitz condition on $g_{j}^{i}$ and $h_{j k}^{i}$. If $w(x)=L x$ on $\mathbb{R}_{+}(L>0$ being a real constant) we obtain from Theorem 2 that

$$
\left\|\left|x_{1}-x_{2}\left\|\left.\right|_{n} ^{2} \leq K_{1} e^{L K_{2}} \mid\right\| \alpha_{1}-\alpha_{2} \|_{n}^{2} .\right.\right.
$$

This proves the Lipschitz character of the solutions with respect to the initial condition in the case of a Lipschitz condition on $g_{j}^{i}$ and $h_{j k}^{i}$.

\section{Parametric dependence of the solution processes on the coefficients}

In this section we consider the problem of the convergence of the solution processes in McShane's stochastic integral equation systems with coefficients depending on a parameter.

Let us consider families of stochastic integral equation systems

$$
\begin{aligned}
x(t)=\alpha_{\lambda}(t) & +\sum_{j=1}^{r} \int_{0}^{t} g_{j, \lambda}(s, x(s)) d z_{j}(s) \\
& +\sum_{j, k=1}^{r} \int_{0}^{t} h_{j k, \lambda}(s, x(s)) d z_{j}(s) d z_{k}(s), \quad 0 \leq t \leq a,
\end{aligned}
$$

where for each $\lambda \in \Lambda, \Lambda$ being an open and bounded subset of $\mathbb{R}^{m}$ $(m \in \mathbb{N})$, the hypotheses $\left(\mathrm{H}_{1}\right)-\left(\mathrm{H}_{4}\right)$ are satisfied. By Theorem 1 we have that for every $\lambda \in \Lambda$, equation (3. $\lambda$ ) has an unique solution $x_{\lambda} \in C[0, a]$.

Let $\lambda_{0}$ be a fixed point in $\Lambda$.

We assume that for every process $x \in C[0, a]$ we have

$$
f_{\lambda}(t, x(t)) \stackrel{P}{\longrightarrow} f_{\lambda_{0}}(t, x(t))
$$


as $\lambda \rightarrow \lambda_{0}$ ( $P$ expresses the convergence in probability) if $f_{\lambda}$ is any one of the functions $g_{j, \lambda}^{i}$ and $h_{j k, \lambda}^{i}$.

By Lemma I and the remark of [2] we have that condition $\left(\mathrm{H}_{5}\right)$ implies that for every $x \in C[0, a]$,

$$
\lim _{\lambda \rightarrow \lambda_{0}} \int_{0}^{a}\left\|f_{\lambda}(s, x(s))-f_{\lambda_{0}}(s, x(s))\right\|^{2} d s=0 .
$$

Theorem 4. If the hypotheses $\left(\mathrm{H}_{1}\right)-\left(\mathrm{H}_{5}\right)$ are satisfied, then $\lim _{\lambda \rightarrow \lambda_{0}}\left\|\mid \alpha_{\lambda}-\alpha_{\lambda_{0}}\right\| \|_{n}=0$ implies $\lim _{\lambda \rightarrow \lambda_{0}}\left\|\left|x_{\lambda}-x_{\lambda_{0}}\right|\right\| \|_{n}=0$.

Proof: The conditions of the theorem allow to state that

$$
\begin{aligned}
& \| x_{\lambda}^{i}(t)- x_{\lambda_{0}}^{i}(t) \|^{2} \leq\left(1+r+r^{2}\right)\left\{\left\|\alpha_{\lambda}^{i}(t)-\alpha_{\lambda_{0}}^{i}(t)\right\|^{2}\right. \\
&+\sum_{j=1}^{r} C^{2} \int_{0}^{t}\left\|g_{j, \lambda}^{i}\left(s, x_{\lambda}(s)\right)-g_{j, \lambda_{0}}^{i}\left(s, x_{\lambda_{0}}(s)\right)\right\|^{2} d s \\
&+\left.\sum_{j, k=1}^{r} C^{2} \int_{0}^{t}\left\|h_{j k, \lambda}^{i}\left(s, x_{\lambda}(s)\right)-h_{j k, \lambda_{0}}^{i}\left(s, x_{\lambda_{0}}(s)\right)\right\|^{2} d s\right\} \\
& \leq\left(1+r+r^{2}\right)\left\{\left\|\alpha_{\lambda}^{i}(t)-\alpha_{\lambda_{0}}^{i}(t)\right\|^{2}\right. \\
&+2 \sum_{j=1}^{r} C^{2} \int_{0}^{t}\left\|g_{j, \lambda}^{i}(s, x(s))-g_{j, \lambda}^{i}\left(s, x_{\lambda_{0}}(s)\right)\right\|^{2} d s \\
&+2 \sum_{j=1}^{r} C^{2} \int_{0}^{t}\left\|g_{j, \lambda}^{i}\left(s x_{\lambda_{0}}(s)\right)-g_{j, \lambda_{0}}^{i}\left(s, x_{\lambda_{0}}(s)\right)\right\|^{2} d s \\
&+2 \sum_{j, k=1}^{r} C^{2} \int_{0}^{t}\left\|h_{j k, \lambda}^{i}(s, x(s))-h_{j k, \lambda}^{i}\left(s, x_{\lambda_{0}}(s)\right)\right\|^{2} d s \\
&\left.+2 \sum_{j, k=1}^{r} C^{2} \int_{0}^{t}\left\|h_{j k, \lambda}^{i}\left(s, x_{\lambda_{0}}(s)\right)-h_{j k, \lambda_{0}}^{i}\left(s, x_{\lambda_{0}}(s)\right)\right\|^{2} d s\right\}, 0 \leq t \leq a,
\end{aligned}
$$


since if $f_{\lambda}$ is any one of the functions $g_{j, \lambda}^{i}, h_{j k, l \lambda}^{i}$ we have

$$
\begin{aligned}
& \int_{0}^{t}\left\|f_{\lambda}\left(s, x_{\lambda}(s)\right)-f_{\lambda_{0}}\left(s, x_{\lambda_{0}}(s)\right)\right\|^{2} d s \\
\leq & \int_{0}^{t}\left(\left\|f_{\lambda}\left(s, x_{\lambda}(s)\right)-f_{\lambda}\left(s, x_{\lambda_{0}}(s)\right)\right\|+\left\|f_{\lambda}\left(s, x_{\lambda_{0}}(s)\right)-f_{\lambda_{0}}\left(s, x_{\lambda_{0}}(s)\right)\right\|\right)^{2} d s \\
\leq & \int_{0}^{t}\left(2\left\|f_{\lambda}\left(s, x_{\lambda}(s)\right)-f_{\lambda}\left(s, x_{\lambda_{0}}(s)\right)\right\|^{2}+2\left\|f_{\lambda}\left(s, x_{\lambda_{0}}(s)\right)-f_{\lambda_{0}}\left(s, x_{\lambda_{0}}(s)\right)\right\|^{2}\right) d s
\end{aligned}
$$

for $0 \leq t \leq a$.

We denote by $J_{1, \lambda}^{i}(t), J_{2, \lambda}^{i}(t), J_{3, \lambda}^{i}(t)$ and $J_{4, \lambda}^{i}(t), 0 \leq t \leq a$, the last four terms in the last member of the previous inequalities.

The hypothesis $\left(\mathrm{H}_{3}\right)$ enables us to state that

$$
\begin{gathered}
\left\|x_{\lambda}^{i}(t)-x_{\lambda_{0}}^{i}(t)\right\|^{2} \leq\left(1+r+r^{2}\right)\left\{\left\|\alpha_{\lambda}^{i}(t)-\alpha_{\lambda_{0}}^{i}(t)\right\|^{2}+J_{2, \lambda}^{i}(t)+J_{4, \lambda}^{i}(t)\right. \\
\left.+2\left(r+r^{2}\right) C^{2} \int_{0}^{t} w\left(\left\|x_{\lambda}(s)-x_{\lambda_{0}}(s)\right\|_{n}^{2}\right) d s\right\}, \quad 0 \leq t \leq a .
\end{gathered}
$$

Denoting

$$
\begin{aligned}
M_{1}(\lambda) & =\left(1+r+r^{2}\right)\left\{\left\|\alpha_{\lambda}-\alpha_{\lambda_{0}}\right\|_{n}^{2}+\sum_{i=1}^{n} J_{2, \lambda}^{i}(a)+\sum_{i=1}^{n} J_{4, \lambda}^{i}(a)\right\} \\
M_{2} & =2 n\left(1+r+r^{2}\right)\left(r+r^{2}\right) C^{2}
\end{aligned}
$$

we see that by the hypothesis $\left(\mathrm{H}_{5}\right)$ we have

$$
\lim _{\lambda \rightarrow \lambda_{0}} \sum_{i=1}^{n}\left(J_{2, \lambda}^{i}(a)+J_{4, \lambda}^{i}(a)\right)=0
$$

and so, since $\lim _{\lambda \rightarrow \lambda_{0}}\left\|\mid \alpha_{\lambda}-\alpha_{\lambda_{0}}\right\| \|_{n}^{2}=0$, we deduce that $\lim _{\lambda \rightarrow \lambda_{0}} M_{1}(\lambda)=0$.

On the other hand, we have that

$$
\left\|x_{\lambda}(t)-x_{\lambda_{0}}(t)\right\|_{n}^{2} \leq M_{1}(\lambda)+M_{2} \int_{0}^{t} w\left(\left\|x_{\lambda}(s)-x_{\lambda_{0}}(s)\right\|_{n}^{2}\right) d s, \quad 0 \leq t \leq a .
$$

Applying Bihari's inequality, we obtain

$$
\left\|x_{\lambda}(t)-x_{\lambda_{0}}(t)\right\|_{n}^{2} \leq G^{-1}\left(G\left(M_{1}(\lambda)\right)+M_{2} t\right), \quad 0 \leq t \leq a,
$$


thus

$$
\left\|\left|x_{\lambda}-x_{\lambda_{0}}\right|\right\|_{n}^{2} \leq G^{-1}\left(G\left(M_{1}(\lambda)\right)+M_{2} a\right) .
$$

Since $\lim _{\lambda \rightarrow \lambda_{0}} M_{1}(\lambda)=0$ we deduce in a similar way to the proof of Theorem 3 that $\left.\lim _{\lambda \rightarrow \lambda_{0}}\left\|x_{\lambda}-x_{\lambda_{0}}\right\|\right|_{n} ^{2}=0$.

Remark 4. The problem of the parametric dependence of the solution processes on the coefficients was studied in [2], in the case of a Lipschitz condition on the functions $g_{j}^{i}$ and $h_{j k}^{i}$.

\section{References}

1. Angulo Ibañez, J. M., Gutiérrez Jaimez, R., On the existence and uniqueness of the solution processes in McShane's stochastic integral equation systems, Ann. Sci. Univ. Blaise Pascal 92 (1988), $1-9$.

2. Angulo Ibañez, J. M., Gutiérrez Jaimez, R., Parametric dependence of the solution processes on the coefficients in McShane's stochastic integral equation systems, Ann. Sci. Univ. Blaise Pascal 92 (1988), 11-22.

3. Bihari, I., A generalization of a lemma of Bellman and its application to uniqueness problems of differential equations, Acta Math. Acad. Sci. Hungar 7 (1956), 71-94.

4. Constantin, A., A Gronwall-like inequality and its applications, Atti Accad. Naz. Lincei Rend. Cl. Sci. Fis. Mat. Natur., Serie IX I, Fasc. 2 (1990), 111-115.

5. Constantin, A., Some observations on a Conti's result, Atti Accad. Naz. Lincei Rend. Cl. Sci. Fis. Mat. Natur., Serie IX II, Fasc. 2 (1991), 137-145.

6. Constantin, A., A random fixed point theorem for multifunctions, Stochastic Anal. Appl. 12(1) (1994), 65-73.

7. Conti, R., Limitazioni' in ampiezza' delle soluzioni di un sistema di equazioni differenziali e applicazioni, Boll. Un. Mat. Ital. 11 (1956), 344-349.

8. Elworthy, K. D., "Stochastic Differential Equations on Manifolds," London Math. Soc. Lecture Notes, Series 70, Cambridge University Press, 1982.

9. LAdDE, G. S., SEIKKala, S., Existence, uniqueness and upper estimates for solutions of McShane type stochastic differential systems, Stochastic Anal. Appl. 4 (1986), 409-430. 
10. McShane, E. J., Stochastic integrals and stochastic functional equations, SIAM J. Appl. Math. 17 (1969), 287-306.

11. McShane, E. J., Toward a stochastic calculus, Proc. Nat. Acad. Sci. U.S.A 63 (1969), 275-280.

12. McShane, E. J., Toward a stochastic calculus, Proc. Nat. Acad. Sci. U.S.A 63 (1969), 1084-1087.

13. McShane, E. J., "Stochastic Calculus and Stochastic Models," Academic Press, New York, 1974.

14. McShane, E. J., Stochastic differential equations, J. Multivariate Analysis 5 (1975), 121-177.

15. Schauder, J., Der Fixpunktsarz in Funktionalräumen, Studia Math. 2 (1930), 171-180.

Courant Institute of Mathematical Sciences

New York University

251 Mercer Street

New York, NY 10012-1185

U.S.A.

Rebut el 15 d'Octubre de 1992 